\title{
The standardisation of due diligence questionnaires: Practical ambition or dream?
}

Received: 26th September, 2005

\section{Steve Butler}

is chief executive of CAMRA Data Services which he jointly established in 2004 with the ambition to be the premier technology provider for consultants and plan sponsors in Europe. Steve has 15 years of experience in senior management positions with leading financial institutions Scottish Widows, AXA and Royal Sun Alliance. Steve holds an MBA from Southampton Business School.

\begin{abstract}
Recent years have seen investment managers completing significantly more diligence questionnaires, putting additional pressure on marketing departments. This paper examines the investment consulting marketplace and the potential for the standardisation of due diligence questionnaires, with the aim of helping asset managers consider what they can best do to maximise their marketing efforts.
\end{abstract}

Keywords: investment manager, investment consultant, RFP, consultant database, manager selection

\section{Introduction}

Over the last five years, investment managers have seen a 70 per cent increase in the number of due diligence questionnaires they have received, which has been exacerbated by a dramatic increase in complexity. This has been driven largely by investment consultants' insatiable desire for information to meet the ever-increasing demands of advising pension funds.

Each investment consultant firm requires information in a different format, requiring asset managers to store and update information in multiple formats.

Steve Butler

CAMRA Data Services

CAMRA House

3 Blackdown Road

Deepcut, Surrey GU16 6SH

UK

Tel: +44 (0)1252 836545 Fax: $+44(0) 1252836366$ Email: steve.butler@ camradata.com
The various combinations of these become very complex and inevitably prone to inaccuracies.

Feedback from investment consultants is that one of the primary issues still facing investment consultants is the timely collection of quality data from investment managers. These issues would suggest that standardisation is a logical development in the industry in order to create efficiencies for asset managers and improve the flow of information to investment consultants.

This standardisation has in part been addressed by the investment consultants, led by the larger consultants who have made data collection a strategic element of their business. Consultants are moving away from issuing a due diligence questionnaire or request for proposal (RFP) for each client manager selection, and favouring the strategic decision to establish a single data repository within their business used by all individuals in the practice. Two technology companies in the UK have taken standardisation further by developing databases for standard due diligence information which are used by multiple investment consultant firms.

This results in a positive effect for 
both investment managers and investment consultants. For asset managers the workload is reduced by the removal of duplicate data requests by the investment consultant, requiring the investment manager to submit only updated information to the investment consultant database. For the investment consultants the data are stored in an electronic database, allowing them to manipulate the data to whatever their required output. However, not all investment consultants have made databases the single source of information within their practice.

The downside for asset managers is that manager selection often takes place without their knowledge, that is until the final stages of selection, when a shortened client-specific RFP is issued, or they are invited to a 'beauty parade'. Thus the asset managers' submission to a consultant database in terms of quality and timeliness is critical to their future new business success. However, as there is no direct link from completing the database to winning new business, asset managers do not always prioritise database completion. Thus the benefits to investment consultants of establishing single databases are being eroded by asset managers. As a result, investment consultants ask for the information again, through RFPs causing duplication and inefficiencies in both the asset managers' and investment consultants' businesses.

\section{Investment consultant marketplace}

To understand the potential for standardisation, in excess of 50 structured qualitative face-to-face interviews with investment consultants in the key developed pension markets across Europe were conducted between 2003 and 2005 . Questioning focused on their interaction with asset managers and their internal processes to manage product and company information before constructing portfolios and selecting managers on behalf of clients. The interviews were not designed to undertake a rigorous empirical analysis of the individual operations of investment consultants; moreover it was broad sweep of the investment consultant community. The main objective was to identify key trends and issues which were preventing them from working efficiently with asset managers and subsequently to advise their clients effectively.

Current value-based marketing strategy has segmentation at its core. Peter Doyle (2000) states 'market segmentation is at the core of all marketing problems. ${ }^{1}$ The task of marketing is to meet the needs of customers. But customers differ in their needs: consequently meeting the needs of customers in a market effectively will require many different types of offer'. In order to fully understand the needs of a very broad group of investment consultants they have been segmented into four groups (see Figure 1):

- consultants using a single proprietary database;

- transitional consultants;

- traditional consultants;

- small innovative consultants.

These groups are not mutually exclusive, but are positioned along a continuum linked predominantly by their size and attention to processes.

\section{Single proprietary database}

These firms are categorised by the fact that they have a single data repository which is accessible by all investment consultants across the firm. Importantly, the database cross-references all data inputs to create a single view of an asset manager. Usually manager or product 
profiles for internal and client use are created and updated directly from the database. These proprietary databases are typically populated from various sources including:

- consultant RFPs;

- consultants' web collection platform;

- Russell/Mellon CAPS AMI;

- CAMRA Asset Manager Database;

- Micropal;

- Moneymate.

Firms in this group are at various stages of development but they all have the short-term strategic vision of a single data platform used by all investment consultants and researchers across their businesses. The motivation to implement this strategy is to remove the manual and labour-intensive use of RFPs in the manager selection process. One of the key characteristics of this segment is that data are collected and analysed in advance of any client manager search, to the extent that prescribed recommendations are often ready and waiting for clients - buy lists. Investment research within this segment is highly structured and process driven. Researchers and investment consultants are organised along a matrix structure, combining the skill sets and knowledge of company specialists and product specialists. Data are analysed with various proprietary processes to identify key managers. When asked the key areas in which asset managers should focus their efforts to develop relationships, the unanimous answer was the timely completion of their database or third-party database from which data was taken. This was identified as an ongoing issue. One stated that it was often quicker to get a response from an RFP rather than waiting for managers to update the database.

Key characteristics:
- single database;

- cross-referencing of data;

- product profiles updated from database;

- do not use RFPs or only use very limited versions;

- structured research teams with product specialists;

- asset manager feedback important;

- do not use asset manager websites;

- database completion key asset manager task.

The asset manager relationship is considered very important to this group with the focus on feedback and open communication with the asset manager. Examples of this commitment are:

- investment consultant training on providing feedback;

- research notes passed to manager for comment;

- database help lines;

- quarterly email news updates;

- annual asset manager meetings;

- communication of ratings.

This group acknowledged the level of effort required by asset managers to complete RFPs. All felt that a qualification process involving the tenders to which they wished to reply is a very positive initiative, in managing both their growth and work capabilities. As this segment only sends out limited or infrequent RFPs, to receive a standard response was acknowledged as very unsatisfactory. This led to the opinion that the asset manager was inexperienced, could not be bothered or was unable to provide the correct data. All of which gave rise to concerns about the suitability of an asset manager. Websites are not often used by this group for the following given reasons:

- managers do not update websites quickly enough; 
- very retail oriented;

- do not provide necessary information;

- too complicated to collect data from every site;

- strategy that their database is the only source of data collection.

Key issues for consultants running a single proprietary database are:

'Biggest problem is asset managers providing standard responses to questionnaires or cut-and-paste answers which are not relevant to the answer required.'

'The biggest problem is that asset managers do not provide sufficient priority to the completion of our database. This often leads to an RFP being sent out because we believe it will generate a quicker response.'

'We still experience problems with certain managers not meeting deadlines for providing data.'

'The principal problem that we face is that there is no one single database. This means collecting data from various sources and in various formats, highlighting consistency and timing issues.'

'Asset managers do not put senior enough resources into providing data for investment consultants.'

'Being told by asset managers that the data are in a consultant database and then finding that they are not.'

\section{Transitional}

The three segments are not all mutually exclusive as all investment consultants are at different stages within their group. This small segment is made up of traditional investment consultants who have established, or are in the process of establishing a single proprietary database which has yet to become part of their business culture. Problems this segment face in the transition from traditional to single proprietary database include:

- timeliness of data input from asset managers.

- internal resources to update information.

- investment consultants using the database as their primary data source.

- investment consultants not prioritising the input of research notes.

- culture change.

This group tends to have characteristics similar to the single proprietary database segment except they are still using RFPs to supplement or support their database. In addition, their investment consultants are not yet in the habit of using the database and will often call asset managers directly to answer questions or supply data, thus creating an element of duplication similar to that of the traditional investment consultants.

In building a relationship with these consultants, the timely completion of their own or third-party databases is equally as important as a quality consultant relationship manager, working with all investment consultants in the practice.

\section{Traditional}

Firms in this segment are predominantly small to medium-sized investment practices operating on a national basis with multiple offices. Their data collection is not as structured as those adopting a single proprietary database strategy: they often have multiple databases supported by multiple data collection efforts. These data collection processes tend to have evolved through the business need for a certain type of data rather than a strategic approach. The processes appear to be effective in isolation but create a high degree of 
duplication across the business. For example, one firm collected information through RFPs to compile product profiles and also collected the same information through RFPs for manager searches, which was also the same information available within a third-party database which they used for ad hoc requests for data. The following data sources were identified:

- consultant RFIs;

- consultant RFPs;

- Russell/Mellon AMI;

- CAMRA Asset Manager Database;

- asset manager internet sites;

- ad hoc requests;

- IPE quest;

- email updates.

Again, firms in this segment are at various stages of development but all firms identified these multiple collection processes as a weakness which was often attributed to limited internal resources. They saw the future in the short to medium term as implementing a single companywide database which was either provided by a third party or developed internally. The research team in this group generally consisted of individuals in combined investment consulting and research roles dedicating between 20-25 per cent of their time to research. This activity would tend to be supported by a small number of data administrators.

Most commonly the researchers were not product specialists but maintained relationships with dedicated asset managers. Of the medium-sized firms in this group, research was reported back to an investment committee which made buy, sell, hold decisions. However, no decisions about actual manager recommendations were made in advance of a manager search. Ultimately, the individual investment consultant within the practice made the decisions regarding the recommendation of managers. In contrast to the single proprietary database group, all of these issues mean that the relationship with the individual investment consultant is critical from both a research and a marketing point of view. Thus a good consultant relationship manager is someone who is able to deal with all information requests, be proactive in providing critical information and have a good working relationship with all individual investment consultants. When asked in which key areas asset managers should focus their efforts to develop their relationships, 100 per cent of firms in this group vigorously stated that a quality relationship manager was essential. Within this segment there was a very reactive attitude towards providing asset manager feedback to manager research visits and business opportunities, as well as updates about the investment consulting business. The most common response was that general feedback would be provided if asset managers asked for it, but it was not always the case that they asked. General comments were:

- do not have the time to spend on this;

- fees are earned providing service to pension funds not asset managers;

- client requests that feedback is not provided;

- not our role to assist asset managers to improve their business.

As a general rule this group is happy for asset managers to qualify whether they want to respond to an RFP, and it was seen as a good thing that asset managers were managing their growth through the tender process. However, there were some concerns that if the qualification was based on size, it could potentially exclude a number of their clients; therefore, they would appreciate asset managers openly discussing this issue 
with them. This group is more open to receiving standard or 'cut-and-paste' answers in RFPs, especially if this improved the response time. They felt that asset managers should be able to identify client-specific questions and that these should be tailored appropriately. As this group does not have a single source of asset manager data within their businesses, they tend to use asset manager websites as one of a number of places where they will attempt to find information. The websites are being used to get product fact sheets, performance data and background information to support reports etc. The Barclays Global Investors (BGI) website was identified as the most useful site which should be used by other asset managers as the benchmark. Ultimately if the website does not meet their needs they resort to contacting the asset manager by telephone. The following general issues were identified as key problems when using websites:

- not updated often enough;

- too much unfocused information making it difficult to find the correct information;

- difficult to easily go to correct information;

- information too general;

- too retail-focused.

Key characteristics:

- multiple databases;

- multiple data collection processes;

- see future in a single database;

- no cross-referencing of data;

- duplication of data requests;

- see a future for full RFPs;

- combined investment consultant and research roles;

- use asset manager websites;

- consultant relationship manager very important;
- want electronic updates from managers.

Comments from this group:

'It is often found that these websites are not updated often enough and it is quicker to get the information direct by telephone from the asset manager.'

'The single most important factor for an asset manager is to provide a quality relationship manager to service our account - someone who is able to identify or collect all relevant information when asked.'

\section{Small innovative}

This segment is made up of small investment consulting firms which are categorised by their entrepreneurial culture and focus on technology. The group is exclusively made up of very experienced investment consultants all with at least 15 years of industry experience. Comparison with a similar sized firm from another segment, they tend to utilise technology solutions or specialists. They have taken their experiences with traditional investment consultancies and identified the shortfalls and inefficiencies within these traditional consulting methods and processes. They have combined this knowledge with technology, either to develop efficient solutions for their own businesses and commercial sale or to become lead users in new industry solutions. Investment consultants in this group split their time between investment consulting and either marketing their technology solutions or promoting them in the marketplace. Examples of these technology solutions are:

- Third-party consultant databases: data collected from asset managers in an electronic database are made available to a number of other investment consultancies. 


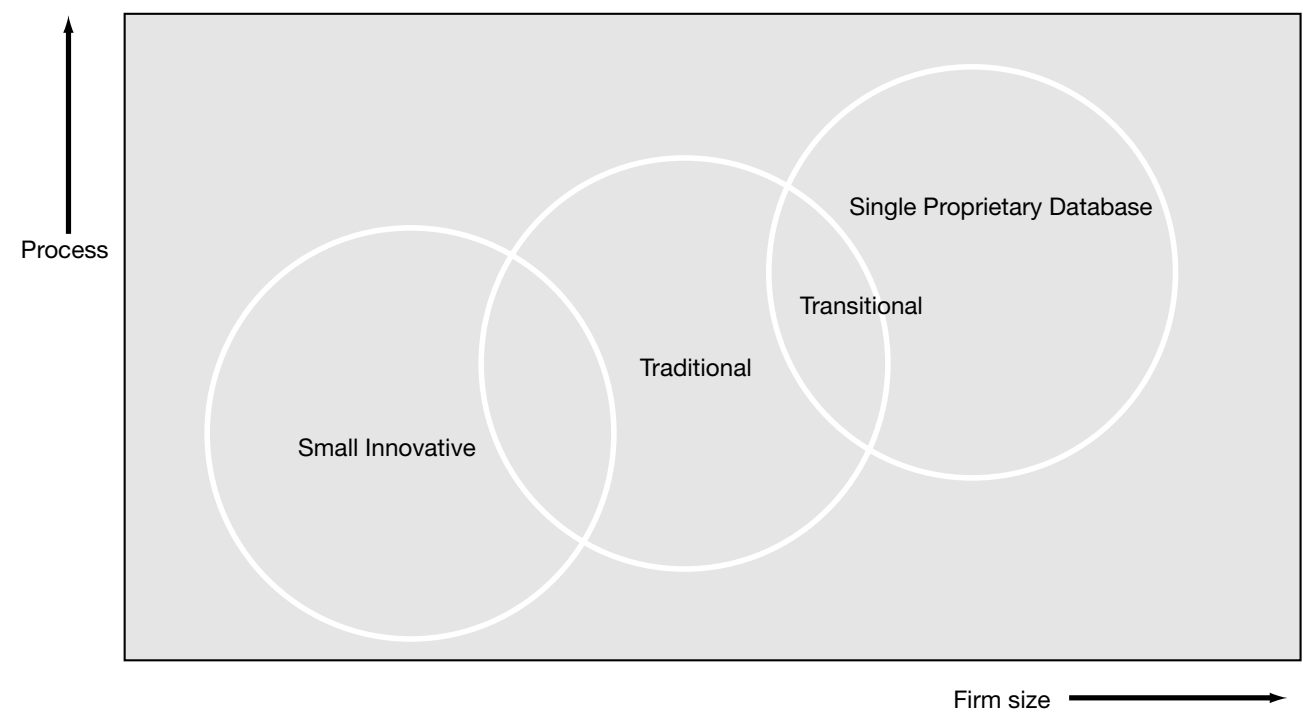

Figure 1: Investment consultant market place

- Pension fund databases: limited versions of the electronic consultant database are made available to pension funds that don't use consultants or want to verify consultant data.

- Electronic tender process: a web questionnaire is distributed to a wide group of asset managers; the responses are then sorted and ranked electronically.

- Public electronic surveys: limited versions of data collected from asset managers in a electronic database are distributed to the public via electronic surveys or websites.

- Pension fund websites: performance data are fed directly into pension fund websites.

'We have developed our process in this way because we believe the onus on asset managers constantly being asked to complete RFPs is not a logical way to do business.'

Key characteristics:

- smaller firms;

- entrepreneurial culture;

- added value through being different;
- high degree of technology usage;

- focus on efficiency;

- attempting to solve issues of traditional consultants.

This segment of investment consultants is problem-solving by nature and is very sympathetic to the workload that asset managers have in responding to RFPs. They require asset managers to have a very open relationship with them, where new ideas and solutions can be discussed and appropriately actioned.

\section{Decline of the RFP}

The research has illustrated a significant growth in consultant databases and their use by investment consultants. Examples of this growth are:

- Consultants who have developed a single proprietary database — this segment has more than doubled in number in the last three years. Among this group several are in the process of developing a web-based interface for asset managers to input and manage their data directly. 
- The existence of a transitional group of investment consultants who are in the process of establishing a single proprietary database.

- The CAMRA Asset Manager Database which provides data to over 350 investment consultants and pension funds was launched online in September 2004. Previously it had been a limited service to a small number of investment consultants receiving data on CD-ROM.

- The launch of dutchinvestor.com in the Netherlands by Bureau Bosch which provides a web-based questionnaire for the Dutch market.

- Expansion of RussellMellon AMI database into the US market.

So, has the issue for asset managers of the volume and complexity of due diligence questionnaires been replaced by volume and complexity of consultant databases? Should the prevailing question be revised to: the standardisation of consultant databases: practical ambition or dream?

Standardisation of a due diligence questionnaire is feasible. It has been done in the hedge fund industry, with the Alternative Investment Managers Association taking the lead when the industry was still in its infancy. However, the standardisation of a myriad of consultant databases developed at significant cost to each investment consulting firm is unlikely. The only situation could be if one of the databases became the dominant provider with other databases joining or disappearing, but this is unlikely due to their proprietary nature.

The research also identified the following reasons:

- The larger investment consulting firms have developed business processes such as research structure and report generation that are based on their current database structure. To change would be complex and costly with relatively insignificant benefit to these firms.

- All investment consulting firms are under constant competitive pressure from their peers and new entrants to the market. This means that questionnaires are constantly evolving to maintain perceived competitive advantage.

- Large investment consultants have developed proprietary databases not only for internal efficiencies but also for marketing their firm's added value to clients. An industry database would remove this added value.

- Local market requirements differ significantly because cultures and markets drive different ways of reviewing and scrutinising investment managers. A recent internal exercise by CAMRA Data Services to consider this issue identified only a 50 per cent overlap between the Investorforce Database (US) and the CAMRA Database (European Database). To meet all local market needs, standardisation would require a database of an overwhelming size, which would probably be unworkable.

- There is a significant number of similar products and services from investment managers and consultants in the pension market; a standard structure would limit a firm's ability to differentiate them. Standardisation would be a logical solution to create efficiencies in the market, but marketing managers from across the industry would vigorously campaign against it.

\section{Solution}

The ultimate solution for standardisation lies in investment managers 
implementing the current emerging technologies which will enable them to automate the distribution of their due diligence information to whoever and in whatever format they require. This will enable investment managers to meet the needs of all segments of investment consultants by providing truly bespoke information to their clients through consultant databases, RFPs, websites or whatever the future holds.

However, for many investment managers it is not that simple. The strong growth rates of the late 1990s established a legacy of standalone business units or merged investment houses operating as a loosely-managed collection of businesses, rather than as a single unit. Correspondingly, these businesses have different processes, systems and reporting standards. As yet there are few (if any) firms who have been able to leverage a broad product range globally across multiple investment processes and accurately present this to investment consultants as a consolidated, compliant and consistent product offering.

In 2002, a group of investment and technology industry professionals were venture capital funded for $\$ 15 \mathrm{~m}$ to establish Activiti. Their brief was to develop a solution using emerging XML and .NET technologies for investment managers to automate the product distribution process. The technology provided a data hub which used consistent information to link directly to consultant databases, create drafts of RFPs and automate the production of fact sheets, client reports and websites. In 2005 they withdrew from the market because investment managers were not ready to implement this level of technology.

Currently there are a handful of small technology companies and consultants working to address the problems investment managers experience in the distribution of due diligence information, but as yet there is no complete provider to enable the investment managers to gain advantage in this area.

\section{Conclusion}

Standardisation of due diligence questionnaires is a logical progression for the investment manager selection process of investment consultants. This has been partly addressed by the investment consultants who have invested in developing online databases to reduce the amount of data requests made upon the investment managers. As a result they have also been able to reorganise their own business processes to create efficiencies.

This trend will mean a reduction in the number of due diligence questionnaires or RFPs that are issued, but from the investment managers' perspective RFPs will be replaced with updates for consultant databases.

Standardisation of consultant databases is unlikely because of the cost and motivation of the investment consultants to make changes. If investment managers are to seek efficiencies in this area, they therefore need to look inward at their business processes and technology. Thus, the next steps for investment managers are:

- Review and prioritise consultant database completion: Assuming that a large number of investment consultants are creating shortlists from information in consultant databases, asset managers need to review all the databases in the market and prioritise the completion of information about key investment products into the appropriate databases. An investment consultant firm that is servicing smaller pension funds may only be interested in UK equity and 
global equity in their database whereas another consultant servicing larger pension clients may also be interested in including Japanese equity or US equity.

- Segment marketing approach to investment consultants: Asset managers need to review segmentation methodology considering whether clients have been segmented by type and geography or segmented by actual client needs. Clients need to be asked what is important to them and how they would like to be supported. Asset managers need to build their marketing strategy around these findings. For example, traditional investment consultants stated that a regularly-updated website was important, while investment consultants with databases stated that updating their database was important. Perhaps investment managers should consider making these two activities part of the same process/task and updating with the same information.

- Review new technologies to automate the distribution of product information: New technologies and business processes are constantly being developed which are either available as services, products or consulting. Perhaps a good starting point would be a small project working with a single database to review how key pieces of data could flow automatically into the database.

\section{Reference}

1 Doyle, P. (2000) 'Value Based Marketing: Marketing Strategies for Corporate Growth', John Wiley, Chichester, UK. 